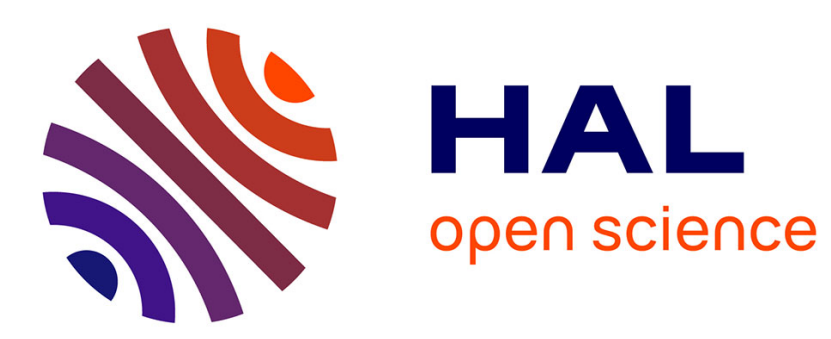

\title{
Entre intégration et patrimonialisation (trans-) frontalières : les dynamiques territoriales de l'ancienne frontière interallemande
}

Christophe Queva

\section{- To cite this version: \\ Christophe Queva. Entre intégration et patrimonialisation (trans-) frontalières : les dynamiques territoriales de l'ancienne frontière interallemande. Cahiers de géographie du Québec, 2012, 56 (159), pp.525-541. 10.7202/1015304ar . halshs-01708858}

\section{HAL Id: halshs-01708858 \\ https://shs.hal.science/halshs-01708858}

Submitted on 14 Feb 2018

HAL is a multi-disciplinary open access archive for the deposit and dissemination of scientific research documents, whether they are published or not. The documents may come from teaching and research institutions in France or abroad, or from public or private research centers.
L'archive ouverte pluridisciplinaire HAL, est destinée au dépôt et à la diffusion de documents scientifiques de niveau recherche, publiés ou non, émanant des établissements d'enseignement et de recherche français ou étrangers, des laboratoires publics ou privés. 


\section{Cahiers de géographie du Québec}

\section{Entre intégration et patrimonialisation (trans-) frontalières : les dynamiques territoriales de l'ancienne frontière interallemande}

\section{Christophe Quéva}

Volume 56, numéro 159, décembre 2012

\author{
URI : id.erudit.org/iderudit/1015304ar \\ DOI : 10.7202/1015304ar
}

Aller au sommaire du numéro

\section{Éditeur(s)}

Département de géographie de l’Université Laval

ISSN 0007-9766 (imprimé)

1708-8968 (numérique)

Découvrir la revue

\section{Résumé de l'article}

RésuméL'ancienne frontière interallemande a longtemps représenté une ligne de fracture majeure au sein des espaces allemand et européen. Avec la réunification de l'Allemagne, elle est passée d'une logique séparative à celle d'une nouvelle interface attractive entre les anciens et les nouveaux Länder. Les enjeux d'intégration ont alors conduit à un gommage certain de la frontière, aussi bien spatialement que politiquement. Néanmoins, l'héritage de la partition reste présent dans les représentations et les pratiques des populations. Il se réaffirme même de façon plus concrète dans le cadre d'un projet de patrimonialisation de tronçons de l'ancienne frontière, dont les enjeux sont à la fois mémoriels et écologiques. En quoi l'ancienne frontière interallemande incarne-t-elle des dynamiques complexes de perméabilité et de «frontiérisation »? En s'appuyant sur l'exemple précurseur du projet de ceinture verte thuringienne, cet article analyse les enjeux spatiaux et identitaires de la patrimonialisation de cette ancienne frontière, de l'échelle locale à l'échelle européenne.

Citer cet article

Quéva, C. (2012). Entre intégration et patrimonialisation (trans-) frontalières : les dynamiques territoriales de l'ancienne frontière interallemande. Cahiers de géographie $d u$ Québec, 56(159), 525-541. doi:10.7202/1015304ar

Tous droits réservés @ Cahiers de géographie du Québec, 2012
Ce document est protégé par la loi sur le droit d'auteur. L'utilisation des services d'Érudit (y compris la reproduction) est assujettie à sa politique d'utilisation que vous pouvez consulter en ligne. [https://apropos.erudit.org/fr/usagers/politiquedutilisation/] 


\title{
Entre intégration et patrimonialisation (trans-) frontalières: les dynamiques territoriales de l'ancienne frontière interallemande
}

\author{
Between integration and (cross-)border \\ patrimonialization: The territorial dynamics \\ of the former inner German border
}

Entre integración y patrimonialización

(trans-) fronterizo: las dinámicas

territoriales de la antigua frontera inter-

alemana

\author{
Christophe QUÉVA \\ Département de géographie \\ Université Paris 1 Panthéon-Sorbonne \\ Christophe.Queva@univ-paris1.fr
}

Résumé

L'ancienne frontière interallemande a longtemps représenté une ligne de fracture majeure au sein des espaces allemand et européen. Avec la réunification de l'Allemagne, elle est passée d'une logique séparative à celle d'une nouvelle interface attractive entre les anciens et les nouveaux Länder. Les enjeux d'intégration ont alors conduit à un gommage certain de la frontière, aussi bien spatialement que politiquement. Néanmoins, l'héritage de la partition reste présent dans les représentations et les pratiques des populations. Il se réaffirme même de façon plus concrète dans le cadre d'un projet de patrimonialisation de tronçons de l'ancienne frontière, dont les enjeux sont à la fois mémoriels et écologiques. En quoi l'ancienne frontière interallemande incarne-t-elle des dynamiques complexes de perméabilité et de «frontiérisation»? En s'appuyant sur l'exemple précurseur du projet de ceinture verte thuringienne, cet article analyse les enjeux spatiaux et identitaires de la patrimonialisation de cette ancienne frontière, de l'échelle locale à l'échelle européenne.

\section{Mots-clés}

Allemagne, frontière, intégration, patrimonialisation, développement local.

\begin{abstract}
For forty years, the Inner German Border represented a major fracture line in German and European political environments. With German reunification, it shifted from a separative function to acting as an interface between the old and the new Länder. Integration tended to be seen as requiring the reality of the border be discounted to better achieve convergence between the physical and political dimensions of the new and previous Länder. However, partition is still widespread in the ways people think and act, and is today even more concretely illustrated in the context of a commemorative and environmentally-driven patrimonialization project for sections of the old border. To what extent does the former Inner German Border embody the complex dynamics of penetrability and frontierization? Drawing on the pioneering example of the "Thuringia Green Belt", this article examines issues of physical space and identity surrounding the patrimonialization project for the former border, at both the local and European levels.
\end{abstract}

\section{Keywords}

Germany, border, integration, patrimonialization, local development. 


\section{Resumen}

La antigua frontera inter-alemana ha representado durante mucho tiempo una línea de fractura mayor en el seno de los espacios alemán y europeo. Con la reunificación alemana ella pasó de una lógica separativa a la de una nueva interfase atractiva entre los antiguos y los nuevos Länder. Entonces, la contingencia de la integración ha conducido a abolir prácticamente la frontera, tanto espacialmente que políticamente. Sin embargo, la herencia de la partición se mantiene en las representaciones y en las prácticas de la población, reafirmándose de manera más concreta dentro de un proyecto de patrimonialización de tramos de la antigua frontera, cuyas consecuencias son a la vez memoriales y ecológicas. ¿De qué manera la antigua frontera inter-alemana encarna dinámicas complejas de permeabilidad y de "fronterización”? Apoyándose en el ejemplo precursor del proyecto de cintura verde de Thuringen, el presente artículo analiza los efectos espacio-identidarios de la patrimonialización de esta antigua frontera, a escala local y a escala europea.

\section{Palabras claves}

Alemania, frontera, integración, partrimonialización, desarrollo local.

\section{Introduction}

L'ancienne frontière interallemande constitue un exemple atypique de discontinuité territoriale héritée: véritable fracture durant les 40 années de séparation entre la République fédérale d'Allemagne (RFA) et la République démocratique allemande (RDA), elle a été progressivement gommée par les dynamiques d'intégration liées à la réunification, tout en laissant subsister un certain nombre de traces. Lacquement l'envisage ainsi «à la fois [comme] modèle et contre-modèle de la géopolitique des frontières» (2002: 9). En tant que modèle, entre 1949 et 1990, elle a joué le rôle de mur - au sens propre comme au figuré - créant une ligne de fragmentation majeure, au sein de l'espace allemand comme de l'espace européen, avec le rideau de fer. En tant que contre-modèle, et ce depuis 1989, elle se caractérise par un processus ambigu de «défonctionnalisation» (Guichonnet et Raffestin, 1974), ayant fait se succéder une phase de perméabilité frontalière quelques mois avant la chute du mur de Berlin, puis une phase d'ouverture de la frontière en 1989-1990, et enfin une phase, plus longue, de gommage de cette dernière depuis 1990. À cet égard, et sur un plan plus théorique, rappelons que, selon la terminologie de Guichonnet et Raffestin (1974), la frontière renvoie à la fois à ligne frontalière (boundary), au sens de limite spatiale de la compétence d'un État et de sa souveraineté territoriale, et à l'espace frontalier (frontier), relevant des sociétés locales qui le pratiquent et se le représentent. Dans ce cadre, la frontière s'associe avant tout à une fonction de contrôle et de surveillance, la «fonctionnalisation » étant un processus de renforcement de la fonction séparative de la frontière. À l'inverse, la défonctionnalisation s'associe à une disparition des fonctions frontalières (Lacquement, 2004), et c'est dans cette perspective que l'ancienne frontière interallemande peut désormais être envisagée comme une nouvelle interface entre les anciens et les nouveaux Länder. 
Néanmoins, le gommage de cette frontière reste partiel, car si celle-ci a effectivement disparu politiquement, des traces frontalières persistent largement, dans les paysages comme dans les pratiques et les représentations des populations. L'ancienne frontière continue ainsi de subsister indirectement ou symboliquement par les discontinuités qu'elle a engendrées sur les plans social et économique entre l'est et l'ouest du pays. Rappelons à cet égard que les espaces bénéficiant ${ }^{1}$, entre 2007 et 2013, de l'objectif européen «Convergence» - destiné aux régions dont le PIB/habitant est inférieur à 75\% de la moyenne communautaire - sont tous situés à l'est de l'Allemagne, entretenant une discontinuité forte et persistante avec l'ouest du pays sur le plan économique. Par ailleurs, plus directement, l'ancienne frontière se voit même réactivée dans le cadre de projets d'aménagement, tendant ainsi à se voir refonctionnalisée sur le plan symbolique en tant que lieu de mémoire et patrimoine national. En effet, elle est devenue, dès les lendemains de la réunification, à la fois le symbole d'une histoire allemande et d'une construction européenne marquées par le poids des fractures et des discontinuités, mais aussi par celui d'une coopération d'acteurs souple et élargie, dans une perspective de protection environnementale. C'est notamment la logique du projet de "ceinture verte» (Grünes Band Deutschland), dont les enjeux sont à la fois mémoriels et écologiques, par la préservation de certains tronçons de l'ancienne frontière.

En quoi l'ancienne frontière interallemande - par l'empreinte territoriale qu'elle a pu laisser, malgré les dynamiques d'intégration liées à la réunification - incarne-telle des dynamiques complexes de perméabilité et de frontiérisation - au sens large de (re)construction frontalière? Plus spécifiquement, dans quelle mesure le projet de patrimonialisation de cette ancienne frontière participe-t-il d'une ambition de refondation territoriale et identitaire en Allemagne, voire dans le cadre européen? Ce projet permet-il l'exploration de nouvelles formes de gouvernance et de coopération d'acteurs, ou entretient-il plutôt une certaine inertie des systèmes d'acteurs? Nous analysons, dans cet article, les dynamiques complexes de déterritorialisation et de reterritorialisation de l'ancienne frontière interallemande. Notre hypothèse est que les dynamiques frontalières héritées relèvent non seulement d'une logique de recomposition territoriale - au sens de reconfiguration de territoires institutionnels associée à des redécoupages territoriaux - mais aussi, et surtout, d'une forme de «refondation territoriale» (Despin, 2003), de «fabrique de territoires» (Giraut, 2005), ceux-ci soient-ils fonctionnels, identitaires ou institutionnels. Pour appréhender les enjeux de cette dynamique frontalière, nous nous appuyons sur l'étude de projets de développement local élaborés autour de l'ancienne frontière dans le Land de Thuringe, notamment sur l'exemple d'un réseau de villes mis en place autour de Sonneberg dans le sud du Land, et sur le projet de ceinture verte de Thuringe (Grünes Band Thüringen). Ces analyses se fondent sur un travail d'entretiens semi-directifs - d'une durée de 45 minutes en moyenne - réalisés entre 2005 et 2007 auprès de 24 acteurs du développement local dans la région de Thuringe du Sud-Ouest (élus, gestionnaires régionaux et socioprofessionnels), ainsi que sur un suivi actualisé des projets, étudiés à travers l'analyse de leurs sites Web ou de la presse locale, notamment le quotidien Südthüringer Zeitung.

1 Il s’agit plus spécifiquement des régions suivantes: Brandebourg Nord-Est, Chemnitz, Dessau, Dresde, Magdebourg, Mecklembourg-Poméranie occidentale et Thuringe. 


\section{Acteurs et vecteurs du gommage de la frontière interallemande}

L'Allemagne est un pays marqué par l'ampleur et la multiplicité des recompositions territoriales, ses frontières politiques externes et internes ayant connu un grand nombre de modifications à toutes les échelles - spatiales et temporelles : «Le sentiment national allemand n'a jamais pu trouver une forme étatique contrairement à l'Angleterre ou à la France. Alors que ces derniers pays ont connu depuis des siècles une formation continue de leur État, l'Allemagne s'est trouvée [...] divisée en de multiples entités politiques» (Foucher, 2005: 12), la division la plus forte et la plus contemporaine correspondant à la création, en 1949, de deux États : la République fédérale d'Allemagne (RFA) et la République démocratique allemande (RDA).

\section{De la séparation à l'unification territoriales}

Ces deux États, incarnant alors deux systèmes politiques et idéologiques antagonistes, se tournaient d'autant plus le dos qu'ils étaient séparés par la partie allemande du rideau de fer - transposé au cœur même de Berlin par le mur construit en 1961. Dans ce contexte, les espaces frontaliers de la RFA et de la RDA correspondaient à des zones globalement répulsives, même si les enjeux d'occupation et de développement étaient totalement différents à l'ouest et à l'est de l'Allemagne. Ainsi, comme avait pu nous l'expliquer un élu investi dans le réseau de villes Sonneberg/Neustadt b. Coburg (figure 1) lors d'un entretien réalisé en 2007, «dans l'ex-RFA, les espaces frontaliers percevaient des subventions. Mais dans l'ex-RDA, c'était plus compliqué... À Sonneberg, on était dans le Sperrgebiet: jusque dans les années 1970, même si on venait de RDA, on avait besoin d'un passeport pour aller voir des gens à Sonneberg» (Entretien, juin 2007, traduction personnelle). En effet, les districts frontaliers de RFA - regroupés dans une zone d'une soixantaine de kilomètres de large nommée le Zonenrandgebiet ${ }^{2}$ (figure 1) - bénéficiaient d'un régime d'aides spécifiques dans le cadre de la politique de soutien aux régions périphériques de frontières (Grenzförderung). En revanche, les espaces frontaliers de la RDA constituaient de véritables marges territoriales sous surveillance militaire, marquées par la présence d'une zone de contrôle et de surveillance (Sperrgebiet) avec, en son sein, une bande de protection de $500 \mathrm{~m}$ de large (Schutzstreifen) grillagée des deux côtés. Enfin, une bande de contrôle (Kontrollstreifen), le long de la frontière, était strictement impénétrable (Lacquement, 2002).

La chute du mur de Berlin en 1989 et la réunification de l'Allemagne en 1990 ont dès lors conduit à la défonctionnalisation de l'ancienne frontière interallemande et à sa disparition a priori totale dans le paysage et dans l'espace: l'Allemagne étant désormais réunifiée, il s'agissait, au début des années 1990, de parvenir à effacer les traces des anciennes divisions, à toutes les échelles territoriales. C'est ainsi que l'organisation fédérale en Länder a été transposée de l'ouest vers l'est, avec la création de six nouveaux Länder sur le territoire de l'ex-RDA, comme on peut le noter sur la figure 1. Par ailleurs, les projets d'infrastructures routières et ferroviaires ont très tôt tenté de reconnecter le réseau de transport largement déséquilibré. Ainsi, le programme de réorganisation des transports pour l'unité allemande (Verkehrsprojekt «Deutsche Einheit») a permis la construction de nouvelles liaisons est-ouest dès 1990-1991 (figure 1).

2 C'est dès 1953 que le Zonenrandgebiet a été défini comme une catégorie spatiale spécifique de la politique d'aménagement en RFA. Dans ce cadre, l'intervention de l'État fédéral dans cet espace a été inscrite dans la loi fondamentale en 1971 (Zonenrandförderungsgesetz). Pour plus de précisions, voir Lacquement (2010). 
Figure 1 La frontière interallemande, entre héritages et gommages frontaliers

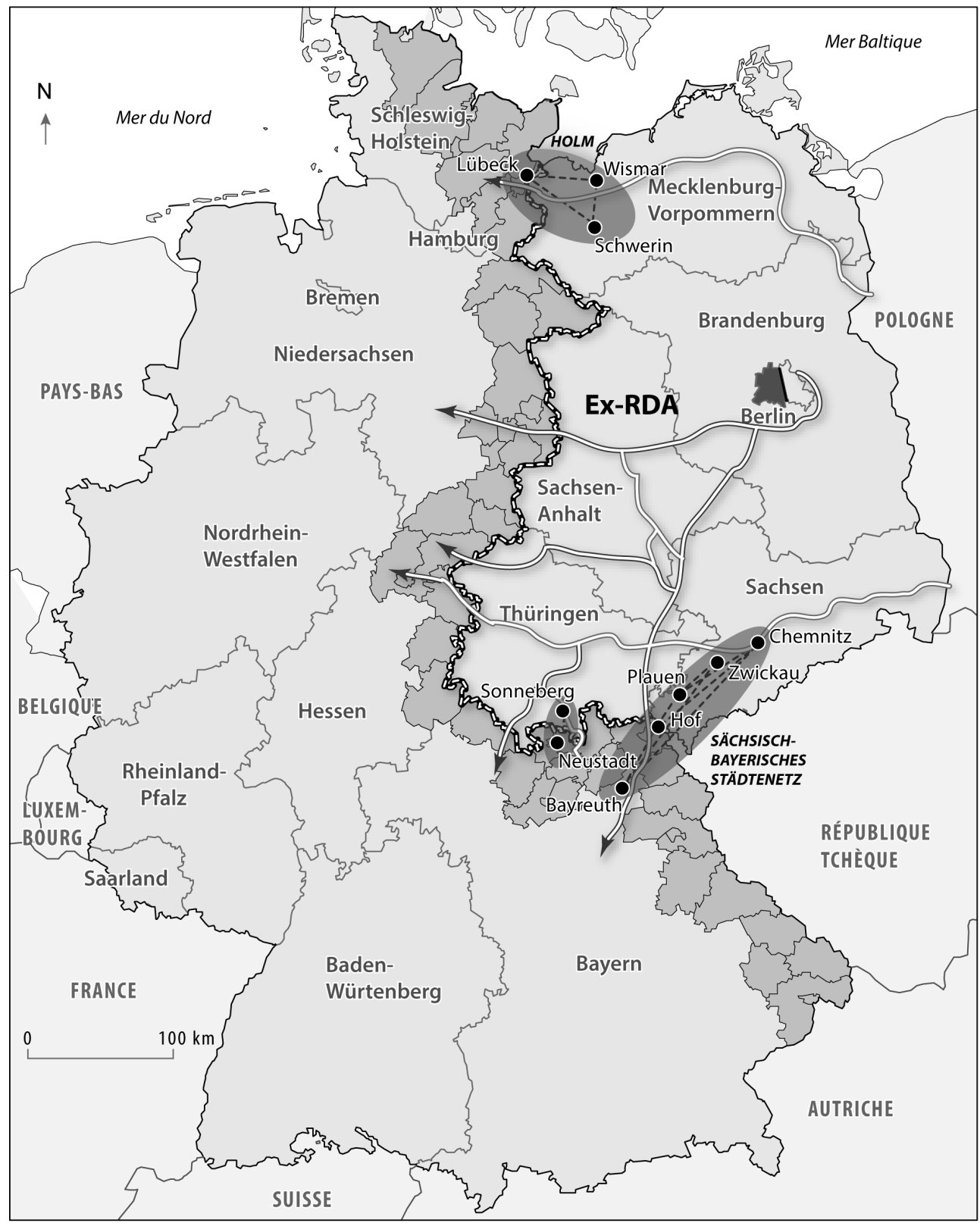

\section{Les héritages frontaliers}

Districts autrefois situés dans le Zonenrandgebiet (RFA)

Zone spéciale de Berlin-Ouest

Ancienne frontière interallemande et Sperrgebiet (RDA)

\section{Vers un gommage de l'ancienne frontière interallemande}

Limites des Länder anciens ( Hessen : ex-RFA) et nouveaux ( Thüringen : ex-RDA)

Nouvelles infrastructures autoroutières issues du programme de transport «Unité Allemande» Verkehrsprojekt «Deutsche Einheit»

Réseaux de villes établis de part et d'autre de l'ancienne frontière 
On peut y lire la volonté nationale non seulement d'arrimer l'est au territoire national, mais aussi de valoriser Berlin - redevenue capitale de l'Allemagne en 1999 - comme nœud de communication. Les enjeux d'intégration ont ainsi conduit à un gommage de la frontière interallemande dans ses dimensions politique et géographique, avec la volonté de relier au maximum les nouveaux et les anciens Länder.

\section{Dépasser les discontinuités: l'exemple des réseaux de villes}

Dans ce cadre, l'ancienne frontière se pose comme le lieu d'une nouvelle coopération entre des acteurs que tout opposait avant la réunification. Les nouveaux liens qui se sont tissés n'ont pas toujours été évidents à mettre en place, en raison du manque d'expérience des acteurs issus de l'ex-RDA en matière d'initiatives locales et de projets de développement. Les nouveaux partenariats établis de part et d'autre de l'ancienne frontière ont néanmoins pu se fonder sur un système de parrainage et sur des stratégies d'accompagnement des nouveaux Länder par les anciens, en particulier pour conduire les réformes territoriales (restauration des Länder à l'est ${ }^{3}$, redécoupage du territoire en districts (Kreise) élargis ${ }^{4}$ : voir les figures 1 et 3 ). Comme l'explique Guder, les enjeux de la disparition de la frontière sont alors l'occasion de repenser les politiques d'aménagement des territoires en Allemagne: «Les années 1990 ont offert de nouveaux défis pour l'aménagement: la réunification allemande a redonné en quelque sorte une nouvelle raison d'être à la politique d'aménagement du territoire. Le problème des disparités régionales et de l'équilibre territorial, notamment entre les nouveaux et les anciens Länder, s'est imposé de nouveau comme sujet d'intérêt politique» (Guder, 2003: 6). C'est ainsi que la réforme de la loi d'aménagement (BauROG) en 1998 a conduit à la mise en œuvre de nouveaux instruments nationaux d'aménagement réseaux de villes, forums régionaux, projets modèles d'aménagement (Modellvorhaben der Raumordnung) - en complément d'une politique gérée précédemment par les Länder. Par ailleurs, cette réforme encourage la mise en œuvre, par les acteurs locaux, de projets de développement local, dans le cadre de coopérations ou de réseaux d'acteurs privés et publics. Cette pensée du réseau est d'ailleurs omniprésente dans les actuelles politiques allemandes d'aménagement (Quéva, 2007). Comme le note le ministère fédéral de l'Aménagement, «la pensée et la planification «réticulaires» prennent une importance croissante dans les pratiques d'aménagement à toutes les échelles» (Bundesministerium für Raumordnung, Bauwesen und Städtebau, 2000, traduction personnelle: 9).

Les réseaux de villes, plus particulièrement, sont l'incarnation même de ces nouvelles modalités d'action. Tout réseau de villes est fondé sur le volontarisme local, autour de projets thématiques tels que le développement économique, le marketing régional (Quéva, 2012), l'amélioration de l'offre touristique, sociale ou encore culturelle. Sur le plan spatial, les réseaux de villes sont assez souvent indépendants des maillages territoriaux. Certains passent ainsi au-delà des limites entre Länder, voire au-delà de

3 Les Länder, qui avaient été supprimés avec la création de la RDA, ont été restaurés en 1990 avec la réunification et l'application, à l'est de l'Allemagne, de l'article 23 de la Loi fondamentale.

4 La réforme territoriale de 1992 a procédé à la fusion des districts dans les nouveaux Länder, afin de les aligner sur le modèle ouest-allemand des districts élargis. On retrouve cette idée à l'échelle des communes avec le modèle de la grande commune fonctionnelle, antithèse de l'émiettement communal. 
l'ancienne frontière interallemande, comme le Sächsisch-Bayerisches Städtenetz (Hof, Bayreuth, Plauen, Chemnitz, Zwickau), le réseau HOLM (Lübeck, Wismar, Schwerin) ou encore le réseau Sonneberg/Neustadt b. Coburg (figure 1). Les deux premiers font partie des réseaux directement soutenus par le gouvernement fédéral comme modèles d'aménagement dans le cadre de la politique Forum Städtenetze (Forum Réseaux de villes) mise en œuvre entre 1998 et 2003, et poursuivie localement par la suite sous la forme de coopérations interurbaines régionales. Le troisième correspond à une coopération locale, non labellisée par le gouvernement fédéral, ce qui ne l'empêche toutefois pas de fonctionner malgré tout, à l'échelle intercommunale, comme nous aurons l'occasion de le préciser par la suite. À chaque fois, l'idée est bien d'accompagner les pratiques transfrontalières des populations, habitant par exemple dans un Land et travaillant dans le Land voisin, et de renforcer les coopérations trans-Länder. Ces mobilités ne cessent d'augmenter autour de l'ancienne frontière interallemande, par exemple en Thuringe, où le pourcentage d'actifs thuringiens travaillant dans un autre Land est passé de 12\% en 1999 à 15\% en 2004 (Thüringer Ministerium für Bau und Verkehr, 2006: 26). Parmi les 122200 Thuringiens concernés à cette date, 91900 - soit 75\% d'entre eux - travaillaient dans l'un des anciens Länder (Hesse et Bavière notamment). Le nombre de navetteurs a par ailleurs augmenté jusqu'en 2008, avant de se stabiliser. Il concerne 127000 personnes en 2010, soit 15,6\% de la population active de Thuringe, d'après les données statistiques du Land de Thuringe publiées en 2011 dans le quotidien Südthüringer Zeitung5.

Dès lors, on pourrait penser que la réunification a conduit à un gommage total de la frontière et même à une intégration transfrontalière, au-delà des limites entre anciens et nouveaux Länder. Or, si «l'Allemagne constitue un observatoire intéressant de dynamiques post-frontalières [...], l'après-frontière invite à cerner des empreintes frontalières, c'est-à-dire la part restante de la limite et de la discontinuité» (Charlot, 2008: 11). Ces empreintes frontalières sont encore largement présentes dans les esprits, parfois même chez les acteurs de l'aménagement, comme l'expliquait en 2005 la représentante du réseau de villes de Thuringe du Sud (coopération intercommunale organisée autour de la petite ville de Suhl, dans le sud du Land de Thuringe): «Il faut encore prendre certaines personnes par la main. Ça fait maintenant presque une génération que les idées d'autonomie et d'indépendance ont fait leur chemin dans les mentalités. Ça a été une difficulté pour tout le monde au début et il faut du temps pour que les gens arrivent à penser différemment: beaucoup, notamment les plus âgés, veulent encore être dirigés» (Entretien, juin 2005, traduction personnelle). Cette idée d'une certaine inertie héritée de la RDA, et à dépasser, mérite toutefois d'être nuancée dans le sens où «l'empreinte frontalière» (Ibid.) est parfois totalement assumée par les acteurs locaux, au point de refonder, symboliquement, la frontière interallemande - cette "frontière invisible», pour reprendre le titre de l'ouvrage de Le Pollotec ${ }^{6}$ (2004).

5 Jolf Schneider, «Jeder sechste Thüringer arbeitet nicht in der Heimat», Südthüringer Zeitung, 12 mars 2011.

6 Notons que, dans cet ouvrage, la frontière est prise en un sens différent, désignant la partition sociale et identitaire entre les Wessis («Ceux de l'ouest») d'une part, et les Ossis («Ceux de l'est»), d'autre part. 


\section{Vers une refondation de la frontière? Les enjeux patrimoniaux de l'ancien espace frontalier}

Si les 20 ans de la chute du mur de Berlin ont été l'occasion de nombreuses célébrations en 2009, en souvenir de cet événement majeur des histoires allemande et européenne, c'est, en 2011, l'anniversaire de la construction du mur qui fait l'objet de commémorations - naturellement moins festives, mais non moins lourdes de sens pour la mémoire allemande. À cet égard, un documentaire sur l'ancienne frontière interallemande a été réalisé par la chaîne de télévision Deutsche Welle (figure 2). Comme l’indique le texte de présentation de ce documentaire, le gommage de la frontière interallemande ne peut être total, en raison d'un impératif mémoriel, quasi identitaire pour le peuple allemand. De l'échelle nationale à l'échelle locale (avec les exemples de Berlin et de Hötensleben), ce documentaire rend compte de l'emprise - spatiale et sociétale - du système frontalier durant les décennies de séparation. De fait, malgré sa disparition au sens politique en 1989-1990, on peut dire que les marques de la frontière restent présentes, non seulement pour une grande partie de la population, mais aussi sur les espaces et les paysages anciennement frontaliers. Ces traces sont d'autant plus visibles dans le contexte post réunification que l'impératif mémoriel se retrouve également dans des opérations de patrimonialisation de certains tronçons de l'ancienne frontière. Cette patrimonialisation peut être individuelle et privée: «Des items résiduels [de la frontière] sont ainsi récupérés par des habitants qui les détournent, des plaques métalliques font office de clôtures privatives de jardins. Cette pratique peut traduire [...] le désir de conserver individuellement un bout de cette histoire collective et l'insertion dans la sphère privée de l'ancien objet ségrégatif et répressif» (Charlot, 2008: 13).

Mais, par ailleurs, la patrimonialisation de la frontière relève également d'une logique collective et d'un vaste projet d'aménagement et de développement local: le projet Grünes Band - projet de ceinture verte à visée mémorielle et écologique (figure 3).

\section{Das Grüne Band: un projet multiscalaire de patrimonialisation de l'ancienne frontière}

Le Grüne Band est un projet de protection de tronçons de l'ancienne frontière interallemande, associant réserves naturelles, chemins de randonnées et lieux de mémoire, qu'il s'agisse de musées de la frontière ou de mémoriaux (figure 3). L'idée d'une «ceinture verte» avait émergé localement dès 1989 dans le Land de Thuringe avec le projet Am Grünen Band Thürigen (Mohnhaupt et Franke, 2004). Elle s'est ensuite développée à l'échelon national, avec le projet Grünes Band Deutschland, formalisé et financé à partir de 2001-2002 par l'État fédéral (Riecken et al., 2009). Enfin, à l'échelle européenne, le projet européen European Greenbelt est inscrit depuis 2004 dans la même logique de patrimonialisation le long de l'ancien rideau de fer, de l'océan Arctique à la mer Noire. La partie allemande ne représente en effet que 1380 des $6800 \mathrm{~km}$ du rideau de fer. Cette ceinture verte européenne, reliant des zones Natura 2000 et soutenue par l'Union internationale pour la conservation de la nature, est le premier réseau écologique transfrontalier traversant toute l'Europe. À cet égard, le projet a reçu en 2010 le Prix Binding pour la conservation de la nature et la protection de l'environnement. Comme on peut le voir sur la figure 3, les 27 lieux de mémoire officiels (anciens miradors, portions de murs, plaques commémoratives, etc.) sont répartis presque tout le long de l'ancienne frontière. 
Figure 2 « Emmurés ! », documentaire sur la frontière interallemande (extraits)
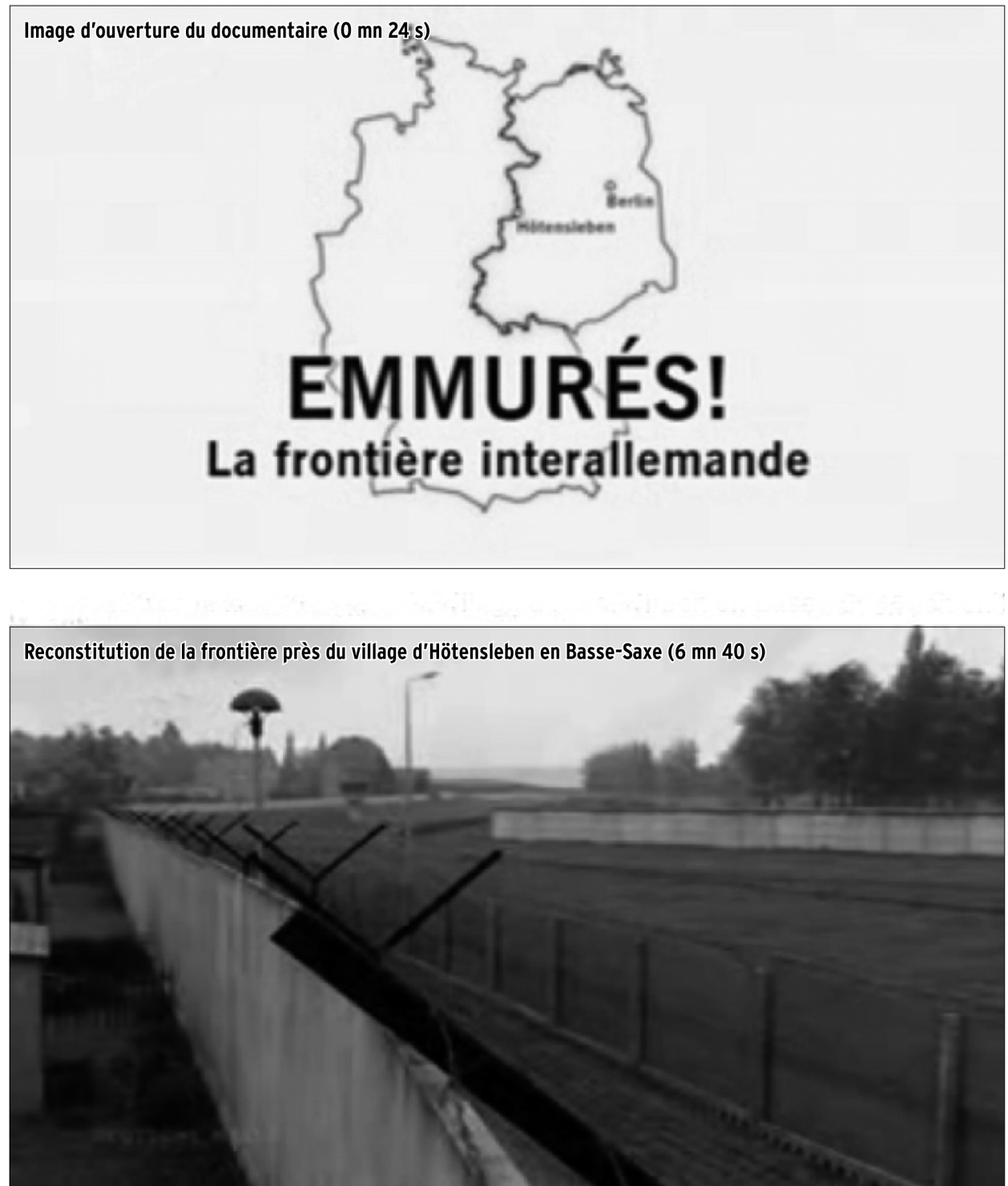

Source: Deutsche Welle, 2009-2011 (Texte de présentation du documentaire sur le site Web de la chaîne : http://www.dw-world.de) Documentaire accessible en ligne (en français)

http://www.dw-world.de/popups/popup_single_mediaplayer/0,4418631_type_video_struct_11820_contentid_442332,00.html

\section{Texte de présentation du documentaire}

"À l'occasion du $20^{e}$ anniversaire de la chute du mur et du 50e anniversaire de sa construction en 2011, la Deutsche Welle a réalisé, en coopération avec la Fondation du mur de Berlin, un projet unique «Emmurés!» - la frontière interallemande. L'animation en $\mathrm{HD}$ du système frontalier à Berlin et de la frontière interallemande révèle l'affligeante brutalité des installations frontalières et répond à la question suivante: "À quoi ressemblait le mur?». Il ne reste que quelques endroits visibles et isolés de la «bande de la mort» et du mur. Mais ces fragments ne permettront pas aux futures générations de comprendre l'horreur et les dangers d'une installation. L'animation HD de la Deutsche Welle permet de mieux l'appréhender et de mieux le comprendre (...). Avec son langage et son attrait visuels, elle est un moyen de toucher les jeunes générations qui considèrent la division de l'Allemagne comme l'histoire passée et qui ne l'ont jamais connue de leur vivant. Elle recrée une atmosphère, celle des souffrances de la population à Berlin et à la frontière interallemande entre la RDA et la République fédérale d'allemagne». 
Figure 3 La refondation patrimoniale de l'ancienne frontière interallemande

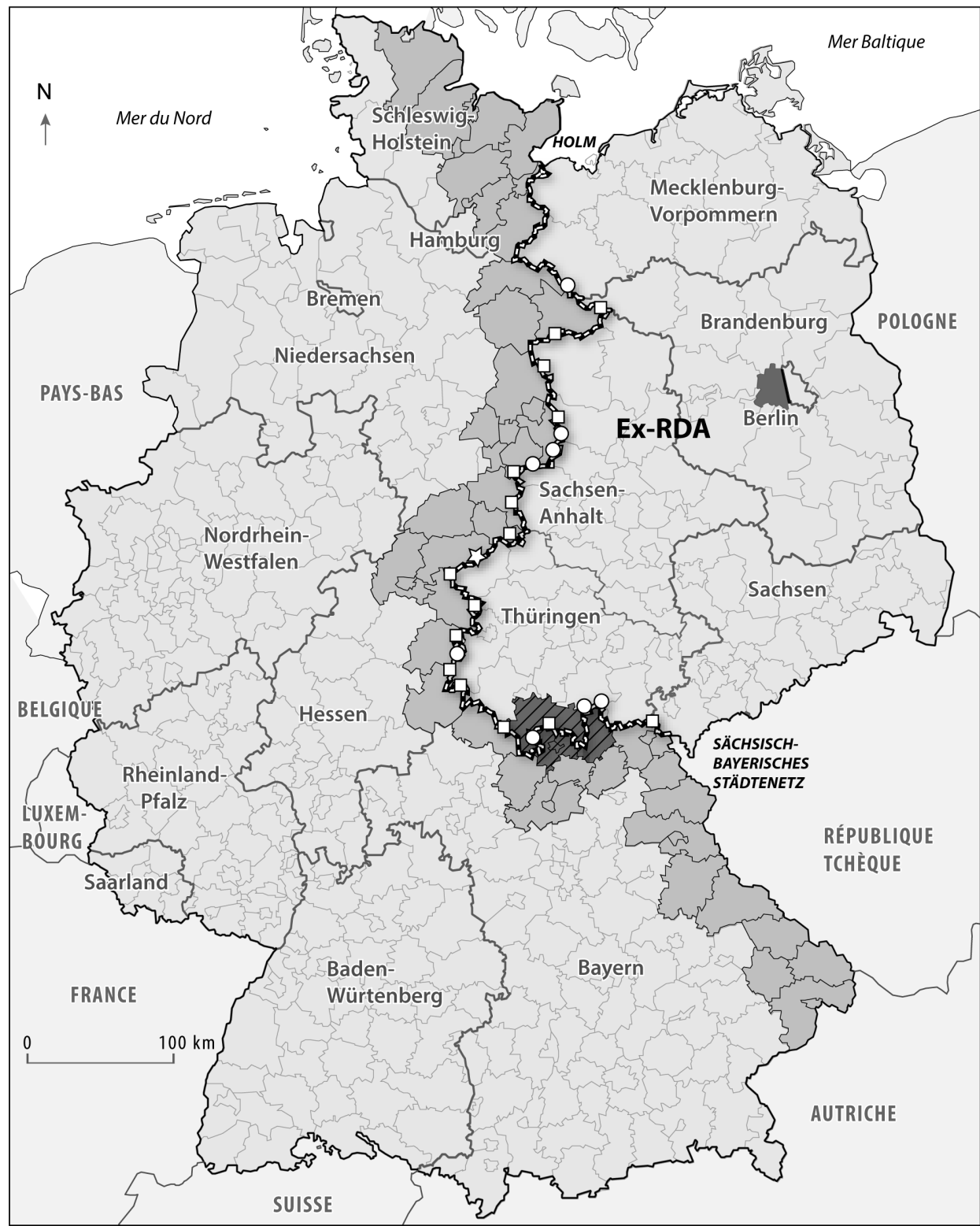

Les héritages frontaliers

Source: Raumordnungsbericht, 2000 ; Deiters J., et al.., 2001 ; Lacquement, 2004.

Districts autrefois situés dans le Zonenrandgebiet (RFA)

Zone spéciale de Berlin-Ouest $\quad$ Ancienne frontière interallemande et Spergebiet (RDA)

\section{Les redécoupages territoriaux : le modèle de la RFA transposé dans l'ex-RDA}

Limites des Länder anciens ( Hessen : ex-RFA) et nouveaux ( Thüringen : ex-RDA)

Limites des districts (Kreise)

\section{L'espace (trans-)frontalier patrimonialisé}

\& Musée de Teistungen

$\square \quad$ Autre musée sur la frontière

- Mémorial de la frontière

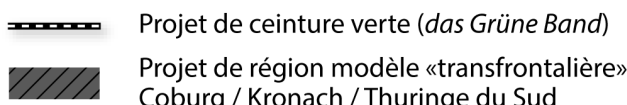


Ces lieux de mémoire se sont mis en place dès les lendemains de la réunification, répondant à une volonté à la fois politique et sociale d'incarner la partition - périodeclef de l'histoire et de l'identité allemandes - dans l'espace et les paysages. Ces «lieux exemplaires» - «élus et produits comme tels» (Bédard, 2002), notamment par les politiques publiques de patrimonialisation - tendent ainsi à relever de plus en plus d'une logique de "haut lieu» - au sens de «lieu localisé et nommé, élevé dans l'échelle des valeurs », s'associant à un signifiant, à un référentiel identitaire et à des "pratiques pérégrinales» (Clerc, 2004). Ainsi, à Teistungen (figure 3), au nord-ouest de la Thuringe, l'ancien point de passage de Duderstadt-Worbis avait été reconverti en musée de la zone frontalière (Grenzlandmuseum) dès 1995. Plus de 15 ans plus tard, ce lieu reste attractif pour le tourisme de mémoire; il a d'ailleurs fait l'objet en 2009-2010 d'un vaste programme de réaménagement destiné à l'agrandir, avec un financement provenant à la fois de l'État fédéral (1 million d'euros), mais aussi des Länder de Thuringe et de Saxe (1,4 million d'euros). L'enjeu patrimonial et mémoriel s'associe ainsi à un réel investissement de la part des acteurs publics.

Par ailleurs, à ces différentes échelles - du local à l'Europe -, la logique est la même : l'absence de fréquentation de l'espace frontalier à l'époque du rideau de fer a paradoxalement conduit à la préservation d'un ensemble faunistique et floristique particulièrement riche et diversifié, sur une bande large de 50 à 200 m correspondant à l'ancienne zone interdite (Sperrgebiet) : «Du point de vue de la protection de l'environnement, l'espace de la frontière intérieure allemande où, sur plus de $1000 \mathrm{~km}$, l'évolution naturelle a pu se dérouler librement (en dehors de l'intense surveillance militaire) constitue un produit dérivé de la division de l'Allemagne pendant des dizaines d'années. Aujourd'hui, des efforts sont consacrés à assurer la protection de la nature dans cet espace» (Chilla, 2007 : 7). Le projet Grünes Band Deutschland se fonde ainsi sur une cartographie précise des zones protégées et sur des moyens financiers issus principalement du ministère fédéral de l'Environnement, de la Protection de la nature et de la Sécurité nucléaire (Schlumprecht et al., 2006). Les acteurs mobilisés aux échelles locale, régionale et nationale relèvent à la fois de la sphère publique (élus locaux, représentants des Länder, du gouvernement fédéral, etc.) et du domaine privé (associations de protection de l'environnement, professionnels du tourisme, etc.), incarnant des formes innovantes de gouvernance et de gestion territoriale contrastant avec les pratiques héritées de la RDA. Des programmes de recherche en environnement sont également mis en œuvre depuis 20 ans pour étudier les dynamiques de cet ancien espace frontalier. On citera notamment le BfN (Bundesamt für Naturschutz) l'Office fédéral pour la protection de la nature - associé à un organisme scientifique, le BUND (Bund für Umwelt und Naturschutz Deutschland e.V.) - l'Organisation pour l'environnement et la protection de la nature.

Cette patrimonialisation mémorielle et environnementale de la frontière relève ainsi d'une logique de refondation territoriale, dans la mesure où c'est un territoire au sens fort qui est recréé par les acteurs investis dans le projet. On pourrait même considérer que cette refondation concerne un territoire non plus seulement frontalier, mais transfrontalier. En effet, ce sont à la fois les anciens et les nouveaux Länder qui s'investissent dans le projet, et non pas uniquement les acteurs de l'ex-RDA. De même, dans son fonctionnement spatial, l'ancienne frontière est dépassée et élargie dans le projet Grüne Band, puisque les parcours de randonnée alternent entre des portions situées à l'est et d'autres à l'ouest de l'Allemagne. Dans cette optique, les 
dynamiques de l'ancienne frontière sont d'autant plus ambiguës qu'au-delà des enjeux de patrimonialisation de certains tronçons, le gommage frontalier se poursuit: "Cette ceinture verte n'est pas une zone de nature dans son intégralité, et seul un tiers est classé zone naturelle protégée. L'association BUND se bat partout où elle le peut, contre une urbanisation galopante » 7 . Entre l'espace de l'intégration et la mémoire de la division, entre la défonctionnalisation et la patrimonialisation de l'ancienne frontière, les enjeux territoriaux de la réunification sont ainsi complexes à appréhender, à l'échelle nationale comme à l'échelle locale.

\section{Entre défonctionnalisation et refondation de l'ancienne frontière: I'exemple de la région de Sonneberg (Thuringe)}

À l'heure actuelle, l'ancienne frontière interallemande présente le paradoxe d'avoir perdu de son épaisseur spatiale tout en conservant une épaisseur symbolique. Du statut de frontière interétatique séparant physiquement deux États avec une zonefrontière étendue et surveillée, elle est certes passée au statut de limite infra-étatique séparant des Länder, autrement dit au statut de découpage administratif. Néanmoins, l'ancienne frontière interallemande reste marquante - au sens fort du terme - dans les paysages, comme on l'a vu, mais aussi dans les pratiques des populations et les stratégies territoriales des acteurs locaux. Ainsi, les différences de niveau de vie, de salaire, de coût du foncier restent encore très importantes entre les anciens et les nouveaux Länder. De ce fait, les choix résidentiels ou d'implantation pour les entreprises relèvent de stratégies complexes jouant sur les différentiels hérités de l'ancienne partition frontalière (Charlot, 2005 ; Roth, 2008). C'est notamment ce que l'on peut noter dans le cas de Sonneberg (Thuringe) et Neustadt b. Coburg (Bavière). Ces deux villes, qui se tournaient autrefois le dos, sont désormais associées dans un réseau de villes (figure 1) destiné à renforcer la coopération en matière de transports en commun et dans le domaine industriel (AG Jürchott-Diener, 2001). En effet, l'ancienne frontière y est devenue une véritable interface, la différence socioéconomique entre la Bavière et la Thuringe entraînant des mobilités de travail entre les deux Länder. Certains des habitants travaillant dans le Land voisin, de nouvelles habitations, généralement des zones pavillonnaires, se sont ainsi développées à proximité immédiate de l'ancienne frontière - là où, du temps de la RDA (en Thuringe actuelle, autrefois dans le Sperrgebiet), le seul fait de se déplacer était interdit. Des entreprises bavaroises ont par ailleurs fait le choix de s'implanter en Thuringe, à proximité de Sonneberg, en raison du faible coût des terrains et des travaux de construction. Les pressions anthropiques sur l'ancienne zone frontalière sont d'autant plus fortes que le réseau de villes Sonneberg/Neustadt b. Coburg est amené depuis peu à s'élargir à une coopération territoriale entre les cinq districts voisins de Sonneberg (Thuringe), Hildburghausen (Thuringe), Coburg (Bavière), Saalfeld (Bavière) et Kronach (Bavière), représentés sur la figure 3. Ce projet de «région modèle» Coburg-Kronach-Thuringe du Sud (Modellregion CoburgKronach-Südthüringen) est porté depuis 2011 par deux acteurs politiques locaux: une élue socialiste au Landtag ${ }^{8}$ de Bavière (SPD), représentante du district de Coburg, et une élue démocrate-chrétienne (CDU), Landrätin ${ }^{9}$ du district de Sonneberg, en

7 Hall, 2008, The Independent, 23 mai 2008 - Traduit en français pour Courrier international.

8 Le Landtag est l'assemblée élue d'un Land, correspondant à son parlement.

9 Le Landrat (féminin: Landrätin) est l'élu local à la tête d'un district (Kreis ou Landkreis). 
Thuringe. Toutes deux défendent actuellement ce projet auprès de leur Länder respectif, tout en cherchant à bénéficier des programmes européens de soutien financier pour la coopération interrégionale. En effet, faire reconnaître la coopération entre les cinq districts précédemment cités comme «région modèle» permettrait d'envisager la mise en œuvre de projets d'aménagement et de développement transfrontaliers, en jouant sur les différences entre la Thuringe et la Bavière: expériences de développement local plus anciennes en Bavière, coût du foncier plus intéressant en Thuringe, etc. Naturellement, ces différences sont également susceptibles d'être associées à un certain nombre de limites, comme le notait dès 2005 l'un des chargés de mission travaillant pour le réseau de villes Sonneberg/Neustadt b. Coburg: «Fonctionnellement, c'est une région économique, mais il reste les frontières entre les Länder. Ce sont deux systèmes différents et les lois posent toujours problème» (Entretien, juin 2005, traduction personnelle).

De fait, au-delà de ces différences - qu'elles soient administratives, économiques ou sociales -, les traces de la frontière persistent dans les paysages ici également. En effet, un chemin de randonnée frontalier (Grenzwanderweg) a été aménagé par les acteurs locaux des districts de Sonneberg et de Coburg, tout en étant par ailleurs directement soutenu financièrement par le Land dans le cadre du projet Grünes Band. La plaquette de présentation du projet dans le district de Sonneberg, et notamment la photographie (figure 4), rendent bien compte de cette «mise en patrimoine» du paysage rural (von Hirschhausen et Lacquement, 2007), en lien avec une valorisation touristique et de loisirs autour de la randonnée pédestre. Cette plaquette - issue d'un document établi par le ministère thuringien de l'Agriculture, de la Protection de la nature et de l'Environnement - évoque l'enjeu touristique représenté par l'ancienne frontière et ses répercussions en termes de développement local; le projet de ceinture verte y est envisagé comme une forme de plus-value territoriale, inscrite dans une perspective de développement durable.

Par ailleurs, d'une manière plus indirecte, la valorisation paysagère et environnementale qui accompagne ce projet est également un vecteur d'attractivité résidentielle à l'échelle locale, dans des espaces marqués par le déclin démographique depuis la réunification. Ainsi, alors que la plupart des régions de l'est de l'Allemagne continuent de perdre des habitants, certaines zones de l'ancienne frontière constituent de nouveaux pôles attractifs, connaissant une croissance de leur population. C'est notamment le cas de la commune de Föritz (3800 habitants), voisine de Sonneberg. Constituée d'un bourg-centre et de 10 localités, qui étaient des communes indépendantes au temps de la RDA, Föritz est aujourd'hui un territoire dynamique, bénéficiant à la fois d'un cadre paysager agréable et de la proximité bavaroise, comme l'indique la fiche de présentation de la commune sur le site Web du district de Sonneberg: "C'est dans un cadre paysager agréable que les anciennes communes indépendantes sont devenues des lieux de vie attrayants, en particulier pour les navetteurs [travaillant en Bavière]. Les localités sont notamment appréciées pour leur caractère villageois » ${ }^{10}$. La constitution de bassins de vie transfrontaliers va même parfois de pair avec une identité renouvelée - inter-Länder voire trans-Länder - comme l'illustre la page Web de présentation du district d'Hildburghausen (voisin de celui de Sonneberg) : «Au sud, les limites du

10 http://www.kreis-sonneberg.de/ (traduction personnelle). 
district sont aussi celles du Land de Bavière, avec lequel se développe un nouveau sentiment d'appartenance commune, en raison des racines historiques de l'identité franconienne, pourtant longtemps divisée par la frontière interallemande»11 12 .

Ainsi, à l'échelle locale, les espaces de l'ancienne frontière interallemande incarnent bien les ambiguïtés de la dynamique (trans-)frontalière qui se jouent à l'heure actuelle. L'ancienne frontière apparaît comme un vecteur de recomposition mais aussi de refondation territoriales, en apparaissant à la fois comme un référentiel mémoriel et comme un différentiel source de nouvelles pratiques et de nouvelles représentations pour les populations et pour les acteurs locaux.

\section{Figure 4 Grüne Band de Thuringe: extraits de la plaquette de présentation}

Extraits de la brochure « Das Grüne Band Thüringen. Ein Projekt zwischen Natur und Geschichte, 2008 ».

Traduction personnelle:

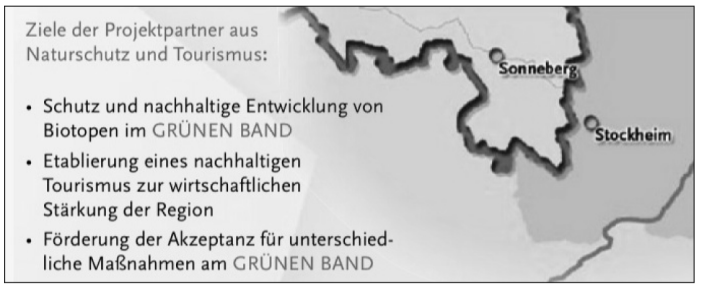

Objectifs des partenaires du projet dans les domaines du tourisme et de la protection de la nature :

-Protéger et valoriser le développement durable des biotopes au sein de la ceinture verte -Mettre en œuvre un tourisme durable pour renforcer l'économie régionale -Favoriser le recours aux différents outils proposés dans le cadre du projet de ceinture verte

\section{Traduction personnelle:}

La ceinture verte dans I'arrondissement de Sonneberg

-Photo: TMLNU (Ministère)

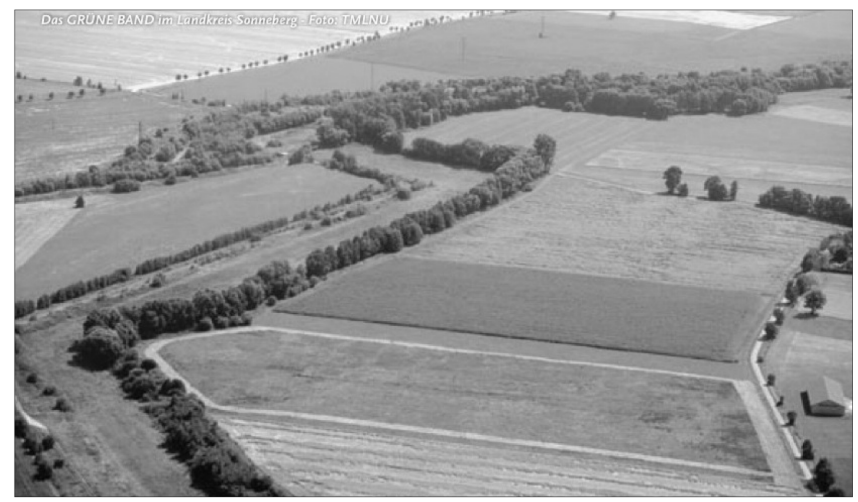

SOUTCe: THÜRINGER MINISTERIUM FÜR LANDWIRTSCHAFT NATURSCHUTZ UND UMWELT, 2008 : p.16 et p.19

11 www.landkreis-hildburghausen.de/index.phtml (traduction personnelle).

12 Principaux sites Webs sur les projets de ceinture verte:

Le chemin de randonnée frontalier dans le district de Sonneberg (Grenzwanderweg im Landkreis Sonneberg) http://www.lkson.de/gbw/

La ceinture verte de Thuringe (Grünes Band Thürigen) http://www.thueringen.de/de/ landentwicklung/aufgaben/gruenes_band/

La ceinture verte allemande (Grünes Band Deutschland) http://www.bfn.de/0310_steckbrief_ gruenesband.html

La ceinture verte européenne (European Green Belt) http://www.greenbelteurope.eu/ 


\section{Conclusion}

De l'échelle locale des sentiers de randonnée pédestre sur la ceinture verte thuringienne (Am Grünen Band Thüringen) à l'échelle européenne des espaces protégés Natura 2000 dans le cadre de l'European Green Belt, en passant par la démarche nationale de ceinture verte allemande (Grünes Band Deutschland), l'ancienne frontière interallemande s'inscrit dans des dynamiques communes de valorisation de la faune et de la flore paradoxalement préservées durant plusieurs décennies dans le cadre d'un rideau de fer quasi impénétrable. La défonctionnalisation de l'ancienne frontière n'est ainsi que partielle: si elle a disparu en tant que frontière interétatique, elle reste un important marqueur symbolique et paysager. De même, dans les pratiques des populations, elle constitue « une nouvelle hétérogénéité spatiale qui perpétue encore aujourd'hui l'existence d'un espace frontalier, au sein duquel les repères territoriaux hérités du temps de la partition jouent un rôle décisif dans l'émergence des nouvelles pratiques spatiales» (Lacquement, 2004: 187). Ces nouvelles pratiques, marquées par la coopération entre les acteurs locaux de part et d'autre de la frontière - que tout opposait par le passé - mobilisent des formes de gouvernance locale qui font de la frontière interallemande un laboratoire particulièrement riche pour analyser les dynamiques d'intégration territoriale postsocialistes. Elles pourraient vraisemblablement trouver un réel écho dans les pays d'Europe centrale et orientale, dans le cadre des expériences de coopération transfrontalière mises en œuvre de part et d'autre de l'ancien rideau de fer. 


\section{Bibliographie}

AG JÜRCHOTT-DIENER (2001) Raumordnerisches Entwicklungskonzept Coburg Dörfles/Esbach - Rödental - Neustadt b. Coburg - Sonneberg. Planungsgruppe Trias.

BÉDARD, Mario (2002) Une typologie du hautlieu, ou la quadrature d'un géosymbole. Cahiers de géographie du Québec, vol.46, $\mathrm{n}^{\circ} 127$, p. 27-48. [En ligne]. www.erudit. org/revue/cgq/2002/v46/n127/023019ar. pdf

BUNDESMINITERIUM FÜR RAUMORDNUNG, BAUWESEN UND STÄDTEBAU (2000) Raumordnungsbericht 2000. Bundesrepublik Deutschland, Selbstverlag.

CHARLOT, Olivier (2008) Après la frontière interallemande. Trajectoires, $\mathrm{n}^{\circ} 2$. [En ligne]. http://trajectoires.revues.org/ index194.html.

CHARLOT, Olivier (2005) Emploi et mobilité dans l'ancienne zone frontière interallemande. Revue d'études comparatives Est-Ouest, vol. 36, n³, p.137-160.

CHILLA, Tobias (2007) La protection de la nature en Allemagne - une nature allemande? Revue Géographique de l'Est, $\mathrm{n}^{\circ} 47$. [En ligne]. http://rge.revues.org/803

CLERC, Pascal (2004) Haut lieu. Hypergeo. [En ligne]. http://www.hypergeo.eu/spip. php?article144

DEITERS, Jürgen, GRÄF, Peter et LÖFFLER, Günter (2001) Nationalatlas - 9. Verkehr und Kommunikation. Leipzig, Institut für Länderkunde - Leibniz Institut.

DESPIN, Laurent (2003) La refondation territoriale. Entre le monde et le lieu. Paris, L'Harmattan.

DEUTSCHE WELLE (2009) Emmurés : la frontière interallemande. Fascicule de présentation du documentaire télévisé, Berlin, DW-Transtel.

FOUCHER, Michel (2005) Espace, frontières et peuplement. Dans Anne-Marie Le Gloannec (dir.) L'Allemagne, peuple et culture. Paris, La Découverte.
GIRAUT, Frédéric (2005) Fabriquer des territoires: utopies, modèles et projets. Paris, Université Paris 1 Panthéon-Sorbonne, Département de Géographie, Thèse d'habilitation à diriger des recherches.

GUDER, Ute (2003) L'aménagement du territoire et la politique régionale en Allemagne: vers une européanisation en douceur? Paris, Groupement d'études et de recherches «Notre Europe».

GUICHONNET, Pierre et RAFFESTIN, Claude (1974) Géographie des frontières. Paris, Presses universitaires de France.

LACQUEMENT, Guillaume (2002) Géographie de la frontière interallemande: recompositions territoriales dans l'ancienne zone frontière interallemande depuis la réunification. Montpellier, UMR 5045 MTE.

LACQUEMENT, Guillaume (2004) Politique d'intégration territoriale et ancienne frontière interallemande. Annales de géographie, $\mathrm{n}^{\circ} 113$, p. 168-187.

LACQUEMENT, Guillaume (2010) Les politiques de développement local à l'épreuve de l'effet de frontière. Jeux d'acteurs et de pouvoirs le long de l'ancienne frontière interallemande. Dans Guillaume Garner, Béatrice von Hirschhausen et Hélène Miard-Delacroix (dir.) Espaces de pouvoir, espaces d'autonomie en Allemagne. Villeneuve d'Ascq, Éditions du Septentrion, p. 179-193.

LE POLLOTEC, Kristel (2004) Allemagne de l'Est: la frontière invisible. Paris, Bartillat.

MOHNHAUPT, Elke et FRANKE, Rainer (2004) Das Grüne Band Thüringen - ein Projekt der Thüringer Landesentwicklungsverwaltung. ZFV, vol.129, n² 2, p. 116-119.

QUÉVA, Christophe (2007) Les paradoxes de la region en Allemagne, entre réseaux et territoires: la région, outil de déterritorialisation? Annales de géographie, $\mathrm{n}^{\circ} 653$, p. 41-64. 
QUÉVA, Christophe (2012) «Marketing régional » et valorisation du rural en Allemagne: discours et argumentaires des acteurs locaux dans la construction de nouveaux territoires. Dans Guillaume Lacquement, Karl Martin Born et Béatrice von Hirschhausen (dir.) Réinventer les campagnes en Allemagne. Paysages, patrimoine et développement local. Lyon, ENS Éditions.

RIECKEN, Uwe et ULLRICH, Karin (2009) Schwerpunkt: Das Grüne Band. 20 Jahre nach dem Fall des Eisernen Vorhangs. Natur und Landschaft, vol. 84, n०9/10, p. 399-403.

ROTH, Hélène (2008) Dynamiques industrielles et mutations des espaces ruraux en Allemagne. Géocarrefour, vol. 83, nº 4 . [En ligne]. http://geocarrefour.revues. org/7035

ROTH, Hélène (2006) Espaces intermédiaires en recomposition. Les campagnes industrielles allemandes en région de frontières. Lyon, ENS Lettres et sciences humaines, thèse de doctorat.

SEDLACEK, Peter (2004) Thüringen - von der Peripherie ins Zentrum? Geographische Rundschau, vol. 56, nº 9, p. 53-57.

SCHLUMPRECHT, Helmut, LUDWIG, Franca, GEIDEZIS, Liawa et FROBEL, Kai (2006) Naturschutzfachliche Schwerpunktgebiete im Grünen Band. Bund Naturschutz in Bayern e.V. (BN) et Landesverband Bayern des Bund für Umwelt und Naturschutz Deutschland e.V. (BUND).

THÜRINGER MINISTERIUM FÜR BAU UND VERKEHR (2006) Demographiebericht, Erfurt, Freistaat Thüringen.

THÜRINGER MINISTERIUM FÜR LANDWIRTSCHAFT NATURSCHUTZ UND UMWELT (2008) Das Grüne Band Thüringen. Ein Projekt zwischen Natur und Geschichte. Erfurt, Freistaat Thüringen.
VON HIRSCHHAUSEN Béatrice et LACQUEMENT, Guillaume (2007) Modèles de l'Ouest, territoires de l'Est: le développement local et la valorisation du patrimoine dans les campagnes d'Allemagne orientale. Revue d'études comparatives Est-Ouest, vol. 38, n³, p. 5-29. 